of injury in England and Wales. By considering a variety of data sources concerning burn injury, the epidemiology can for the first time be accurately be described for the entire population of England and Wales. By analysing and extrapolating representative information from regionally held Emergency Department data sets, adding these to National Health Service Hospital Episode Statistics (NHS.HES) data (1990 to 2009 $225 \mathrm{~K}$ cases), and most crucially data (2003-2009 25K cases) from the International Burn Injury Database (www.iBIDb.org) a full picture has emerged. Analysis has allowed cross validation of capture rates between NHS and iBID data sets and has allowed estimates of the injury load down to small geographical areas (lower super output areas). For rare severe injuries, particularly in children, larger catchment groupings are more appropriate (postcode district) and reveal wide variations in injury incidence rates, particularly in paediatric age groups. Similarly the modes of injury differ both geographically and over time. This complex interrelation will be the subject of the presentation.

\title{
0753 THE EPIDEMIOLOGY AND CONTINUED MONITORING OF BURN INJURY IN ENGLAND AND WALES
}

K Dunn* Correspondence: International Burn Injury Database, Burn Centre, Acute Block UHSM Southmoor Road, Manchester M23 9LT, UK

10.1136/ip.2010.029215.753

In order to prevent injury in a large population one must first understand the distribution geographically, across social and age groups and analyse the differing forms of causation and severity. Not simply as a snapshot but the variation of these variables over time. Continuing analysis is imperative to assess the impact of any prevention methodologies employed. For the first time, these variables can be described for at least one form 